\title{
Magnetic corrections to $\pi-\pi$ scattering lengths in the linear sigma model
}

\author{
M. Loewe, ${ }^{1,2,3, *}$ L. Monje, ${ }^{1, \dagger}$ and R. Zamora, ${ }^{4,5,}$ \\ ${ }^{1}$ Instituto de Física, Pontificia Universidad Católica de Chile, Casilla 306, Santiago 22, Chile \\ ${ }^{2}$ Centre for Theoretical and Mathematical Physics and Department of Physics, \\ University of Cape Town, Rondebosch 7700, South Africa \\ ${ }^{3}$ Centro Científico Tecnológico de Valparaíso-CCTVAL, Universidad Técnica Federico Santa María, \\ Casilla 110-V, Vaparaíso, Chile \\ ${ }^{4}$ Instituto de Ciencias Básicas, Universidad Diego Portales, Casilla 298-V, Santiago, Chile \\ ${ }^{5}$ Centro de Investigación y Desarrollo de Ciencias Aeroespaciales (CIDCA), \\ Fuerza Aérea de Chile, Santiago 8020744, Chile
}

(Received 5 January 2018; published 27 March 2018)

\begin{abstract}
In this article, we consider the magnetic corrections to $\pi$ - $\pi$ scattering lengths in the frame of the linear sigma model. For this, we consider all the one-loop corrections in the $s, t$, and $u$ channels, associated to the insertion of a Schwinger propagator for charged pions, working in the region of small values of the magnetic field. Our calculation relies on an appropriate expansion for the propagator. It turns out that the leading scattering length, $l=0$ in the $S$ channel, increases for an increasing value of the magnetic field, in the isospin $I=2$ case, whereas the opposite effect is found for the $I=0$ case. The isospin symmetry is valid because the insertion of the magnetic field occurs through the absolute value of the electric charges. The channel $I=1$ does not receive any corrections. These results, for the channels $I=0$ and $I=2$, are opposite with respect to the thermal corrections found previously in the literature.
\end{abstract}

DOI: 10.1103/PhysRevD.97.056023

\section{INTRODUCTION}

Scattering lengths were introduced a long time ago in nuclear physics as an important quantity in order to calculate low-energy interactions between nucleons and also in pion-nucleon systems. The scattering lengths of two-pion systems are relevant in order to explore QCD predictions in the low-energy sector. They were first measured by Rosellet et al. [1]. New measurements have been reported using the formation of pionium atoms in the DIRAC experiment [2], establishing for the S-wave $\pi-\pi$ scattering lengths a $4 \%$ difference between scattering lengths in the isospin channels $I=0$ and $I=2$. Another experimental measurement can be explored in the heavy quarkonium $\pi^{0}-\pi^{0}$ transitions [3]. In the past, thermal effects on scattering lengths have been considered by many authors in the literature, invoking effective approaches as the Nambu-Jona-Lasinio model [4] or the linear sigma model [5]. A common result, at least qualitatively, is that

\footnotetext{
*mloewe@fis.puc.cl

†nmonje@uc.cl

rzamorajofre@gmail.com
}

Published by the American Physical Society under the terms of the Creative Commons Attribution 4.0 International license. Further distribution of this work must maintain attribution to the author(s) and the published article's title, journal citation, and DOI. Funded by SCOAP ${ }^{3}$. the projection of the scattering lengths in the isospin $I=0$ channel grows, whereas it diminishes in the $I=2$ channel for an increasing temperature.

In peripheral heavy ion collisions, huge magnetic fields appear. In fact, the biggest fields existing in nature. The interaction between the produced pions in those collision may be strongly affected by the magnetic field. In this article, we analyze, in the frame of the linear sigma model, the influence of the magnetic field on the $\pi$ - $\pi$ scattering lengths. For this purpose, we will use the weak field expansion of the bosonic Schwinger propagator [6]. We present in detail the different analytical techniques we have used for our calculations.

\section{LINEAR SIGMA MODEL AND $\pi-\pi$ SCATTERING}

The linear sigma model was introduced by Gell-Mann and Lévy [7] as an effective approach for describing chiral symmetry breaking via explicit and spontaneous mechanism. In the phase in which the chiral symmetry is broken, the model is given by

$$
\begin{aligned}
\mathcal{L}= & \bar{\psi}\left[i \gamma^{\mu} \partial_{\mu}-m_{\psi}-g\left(s+i \vec{\pi} \cdot \vec{\tau} \gamma_{5}\right)\right] \psi \\
& +\frac{1}{2}\left[(\partial \vec{\pi})^{2}+m_{\pi}^{2} \vec{\pi}^{2}\right]+\frac{1}{2}\left[(\partial \sigma)^{2}+m_{\sigma}^{2} s^{2}\right] \\
& -\lambda^{2} v s\left(s^{2}+\vec{\pi}^{2}\right)-\frac{\lambda^{2}}{4}\left(s^{2}+\vec{\pi}^{2}\right)^{2}+\left(\varepsilon c-v m_{\pi}^{2}\right) s .
\end{aligned}
$$


In this expression, $v=\langle\sigma\rangle$ is the vacuum expectation value of the scalar field $\sigma$. The idea is to define a new field $s$ such that $\sigma=s+v$. Obviously, $\langle s\rangle=0 . \psi$ corresponds to an isospin doublet associated to the nucleons, $\vec{\pi}$ denotes the pion isotriplet field, and $c \sigma$ is the term that breaks explicitly the $S U(2) \times S U(2)$ chiral symmetry. $\epsilon$ is a small dimensionless parameter. It is interesting to remark that all fields in the model have masses determined by $v$. In fact, the following relations are valid: $m_{\psi}=g v, m_{\pi}^{2}=\mu^{2}+\lambda^{2} v^{2}$, and $m_{\sigma}^{2}=$ $\mu^{2}+3 \lambda^{2} v^{2}$. Perturbation theory at the tree level allows us to identify the pion decay constants as $f_{\pi}=v$. This model has been considered in the context of finite temperature by several authors, discussing the thermal evolution of masses, $f_{\pi}(T)$, the effective potential, etc. [8-15].

Since our idea is to use the linear sigma model for calculating $\pi-\pi$ scattering lengths, let us remind the reader briefly of the formalism. A scattering amplitude has the general form $[16,17]$

$$
\begin{aligned}
T_{\alpha \beta ; \delta \gamma}= & A(s, t, u) \delta_{\alpha \beta} \delta_{\delta \gamma}+A(t, s, u) \delta_{\alpha \gamma} \delta_{\beta \delta} \\
& +A(u, t, s) \delta_{\alpha \delta} \delta_{\beta \gamma},
\end{aligned}
$$

where $\alpha, \beta, \gamma$, and $\delta$ denote isospin components.

By using appropriate projection operators, it is possible to find the isospin-dependent scattering amplitudes

$$
\begin{gathered}
T^{0}=3 A(s, t, u)+A(t, s, u)+A(u, t, s) \\
T^{1}=A(t, s, u)-A(u, t, s), \\
T^{2}=A(t, s, u)+A(u, t, s),
\end{gathered}
$$

where $T^{I}$ denotes a scattering amplitude in a given isospin channel.

As is well known [16], the isospin-dependent scattering amplitude can be expanded in partial waves $T_{l}^{I}$,

$$
T_{\ell}^{I}(s)=\frac{1}{64 \pi} \int_{-1}^{1} d(\cos \theta) P_{\ell}(\cos \theta) T^{I}(s, t, u) .
$$

Below the inelastic threshold, the partial scattering amplitudes can be parametrized as [18]

$$
T_{\ell}^{I}=\left(\frac{s}{s-4 m \pi^{2}}\right)^{\frac{1}{2}} \frac{1}{2 i}\left(e^{2 i \delta_{\ell}^{I}(s)}-1\right),
$$

where $\delta_{\ell}$ is a phase shift in the $\ell$ channel. The scattering lengths are important parameters in order to describe lowenergy interactions. In fact, our last expression can be expanded according to

$$
\Re\left(T_{\ell}^{I}\right)=\left(\frac{p^{2}}{m_{\pi}^{2}}\right)^{\ell}\left(a_{\ell}^{I}+\frac{p^{2}}{m_{\pi}^{2}} b_{\ell}^{I}+\cdots\right) .
$$

The parameters $a_{\ell}^{I}$ and $b_{\ell}^{I}$ are the scattering lengths and scattering slopes, respectively. In general, the scattering lengths obey $\left|a_{0}\right|>\left|a_{1}\right|>\left|a_{2}\right| \ldots$ If we are only interested in the scattering lengths $a_{0}^{I}$, it is enough to calculate the scattering amplitude $T^{I}$ in the static limit, i.e., when $s \rightarrow 4 m_{\pi}^{2}, t \rightarrow 0$, and $u \rightarrow 0$ :

$$
a_{0}^{I}=\frac{1}{32 \pi} T^{I}\left(s \rightarrow 4 m_{\pi}^{2}, t \rightarrow 0, u \rightarrow 0\right) .
$$

\section{ONE-LOOP MAGNETIC CORRECTIONS FOR $\pi-\pi$ SCATTERING LENGTHS}

The tree-level diagrams shown in Fig. 1, where the continuum line denotes a pion and the dashed line denotes a sigma meson, contribute to the $\pi-\pi$ scattering amplitude. The diagram with a sigma exchanged meson has to be considered also in the crossed $t$ and $u$ channels. From these diagrams, it is possible to get the results shown in Table I. The isospin-dependent scattering amplitudes at tree level have the form

$$
\begin{gathered}
T^{0}(s, t, u)=-10 \lambda^{2}-\frac{12 \lambda^{4} v^{2}}{s-m_{\sigma}^{2}}-\frac{4 \lambda^{4} v^{2}}{t-m_{\sigma}^{2}}-\frac{4 \lambda^{4} v^{2}}{u-m_{\sigma}^{2}}, \\
T^{1}(s, t, u)=\frac{4 \lambda^{4} v^{2}}{u-m_{\sigma}^{2}}-\frac{4 \lambda^{4} v^{2}}{t-m_{\sigma}^{2}}, \\
T^{2}(s, t, u)=-4 \lambda^{2}-\frac{4 \lambda^{4} v^{2}}{t-m_{\sigma}^{2}}-\frac{4 \lambda^{4} v^{2}}{u-m_{\sigma}^{2}} .
\end{gathered}
$$

Note that the linear sigma model is in better agreement at tree level with the experimental results than first-order chiral perturbation theory.

The magnetic corrections to the scattering lengths will be calculated using an appropriate expansion of the Schwinger bosonic propagator, which is given by

$$
i \Delta(k)=\int_{0}^{\infty} \frac{d s}{\cos (q B s)} e^{i s\left(k_{\|}^{2}-k_{\perp}^{2} \frac{\tan (q B s)}{q B s}-m_{\pi}^{2}+i \epsilon\right)} .
$$

In the above expression, $k_{\|}$and $k_{\perp}$ represent the parallel and perpendicular components of the momentum $k$ with respect to the external magnetic field $B$. In general, as is well known, this propagator includes a phase factor, which, however, does not play any role in our calculation. We proceed by taking the weak field expansion [6]
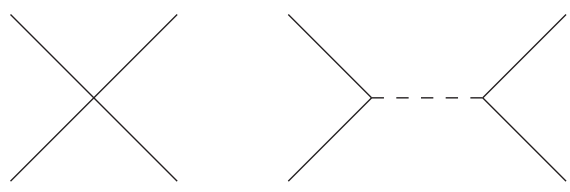

FIG. 1. Tree-level diagrams. 
TABLE I. Comparison between the experimental values [19], first-order prediction from chiral perturbation theory [20], and our results at tree level.

\begin{tabular}{cccc}
\hline \hline & $\begin{array}{c}\text { Experimental } \\
\text { results }\end{array}$ & $\begin{array}{c}\text { Chiral perturbation } \\
\text { theory }\end{array}$ & $\begin{array}{c}\text { Linear sigma } \\
\text { model }\end{array}$ \\
\hline$a_{0}^{0}$ & $0.218 \pm 0.02$ & $\frac{7 m_{\pi}^{2}}{32 \pi f_{\pi}^{2}}=0.16$ & $\frac{10 m_{\pi}^{2}}{32 \pi f_{\pi}^{2}}=0.22$ \\
$b_{0}^{0}$ & $0.25 \pm 0.03$ & $\frac{m_{\pi}^{2}}{4 \pi f_{\pi}^{2}}=0.18$ & $\frac{49 m_{\pi}^{2}}{128 \pi f_{\pi}^{2}}=0.27$ \\
$a_{0}^{2}$ & $-0.0457 \pm 0.0125$ & $\frac{-m_{\pi}^{2}}{16 \pi f_{\pi}^{2}}=-0.044$ & $\frac{-m_{\pi}^{2}}{16 \pi f_{\pi}^{2}}=-0.044$ \\
$b_{0}^{2}$ & $-0.082 \pm 0.008$ & $\frac{-m_{\pi}^{2}}{8 \pi f_{\pi}^{2}}=-0.089$ & $\frac{-m_{\pi}^{2}}{8 \pi f_{\pi}^{2}}=-0.089$ \\
$a_{1}^{1}$ & $0.038 \pm 0.002$ & $\frac{m_{\pi}^{2}}{24 \pi f_{\pi}^{2}}=0.030$ & $\frac{m_{\pi}^{2}}{24 \pi f_{\pi}^{2}}=0.030$ \\
$b_{1}^{1}$ & $\ldots$ & 0 & $\frac{m_{\pi}^{2}}{48 \pi f_{\pi}^{2}}=0.015$ \\
\hline \hline
\end{tabular}

$$
\begin{aligned}
i \Delta(k)= & \frac{i}{k^{2}-m_{\pi}^{2}+i \epsilon}-\frac{i(q B)^{2}}{\left(k^{2}-m_{\pi}^{2}+i \epsilon\right)^{3}} \\
& -\frac{2 i(q B)^{2} k_{\perp}^{2}}{\left(k^{2}-m_{\pi}^{2}+i \epsilon\right)^{4}} .
\end{aligned}
$$

Certainly, only the charged pions will receive a magnetic correction. There are many diagrams that contribute to the pion-pion scattering amplitude at the one-loop level. For each one of these diagrams, we have to add also the corresponding crossed $t$ - and $u$-channel diagrams. In Fig. 2, we have shown only the $s$-channel contribution.

Explicit expressions will be given only for diagram (a) and its equivalent diagram in the $t$ channel. When it corresponds, symmetry factors, isospin index contractions, and multiplicity factors should be included. We work in the center-of-mass momentum $p=\left(2 m_{\pi}, \overrightarrow{0}\right)$. For our calculation, since the sigma meson has a much bigger mass than the pions, its propagator will be contracted to a point, the so-called high mass limit. A numerical treatment, however, confirms the validity of such approximation in our case. For diagram (a) in Fig. 2, we have

$$
\begin{aligned}
i M_{a, s}= & -2 \lambda^{4} I_{a} \int \frac{d^{4} k}{(2 \pi)^{4}} i \Delta\left(k_{0}, \vec{k}, m_{\pi}\right) \\
& \times i \Delta\left(k_{0}-2 m_{\pi}, \vec{k}, m_{\pi}\right) .
\end{aligned}
$$

The corresponding $t$-channel diagram in Fig. 3, where no external momentum flows through the loop, is given by

$$
i M_{a, t}=-4 \lambda^{4} I_{a} \int \frac{d^{4} k}{(2 \pi)^{4}}\left[i \Delta\left(k_{0}, \vec{k}, m_{\pi}\right)\right]^{2},
$$

where $I_{a}$ is an isospin term associated to this diagram for the corresponding channel, which emerges from the contraction of the external pion isospin indices, which is given by

$$
I_{a}=\left(7 \delta_{\alpha \beta} \delta_{\gamma \delta}+2 \delta_{\alpha \gamma} \delta_{\beta \delta}+2 \delta_{\alpha \delta} \delta_{\beta \gamma}\right) \text {, }
$$

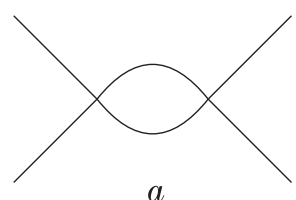

$a$
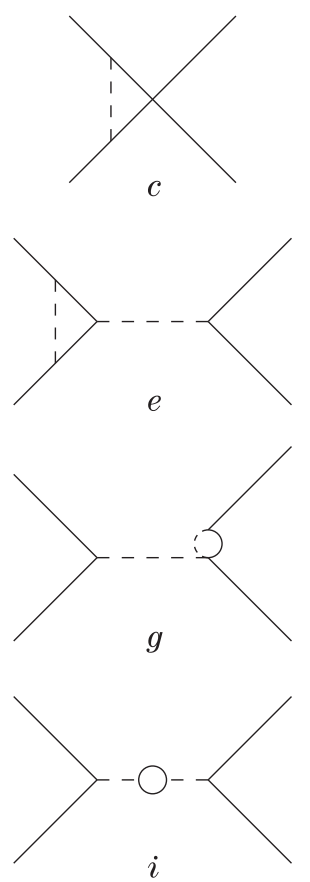

$i$

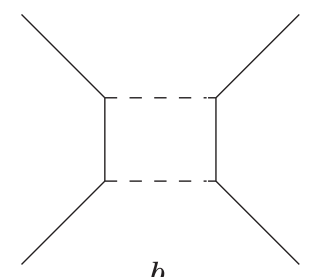

$b$
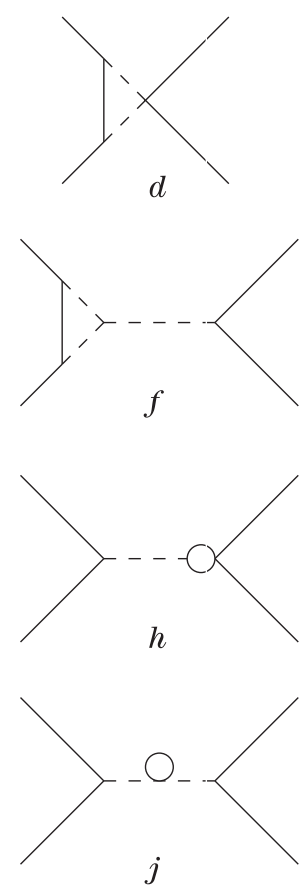

$j$
FIG. 2. Relevant one-loop diagrams in the $s$ channel.

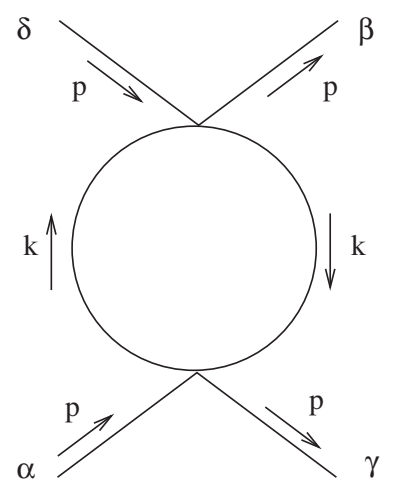

FIG. 3. Diagram (a) for the corresponding $t$ channel.

where the greek letters denote isospin indices. To get the scattering lengths in the different isospin channels, we have used appropriate projectors, contracting them with the amplitudes [see Eq. (2)] that emerged from our calculation.

Notice that for the determination of the scattering lengths we only need the imaginary part of our diagrams. It is natural in this context to choose the $\pi$ - $\pi$ center-of-mass frame of reference for carrying on the calculations in the $s$ channel. In fact, the scattering lengths are defined in this 
frame. Let us consider first the $s$-channel diagram (a). The idea is to calculate the loop using the weak expansion for the propagators. Then, we get expressions that involve free propagators or powers of them. We found it quite useful to use the following technique, introduced in Ref. [21], where a proper time representation for the free propagators is used. The one-loop diagram, at the lowest order, i.e., using normal free propagators

$$
i L(p)=\int \frac{d^{4} k}{(2 \pi)^{4}} D(k) D(p-k),
$$

can be written in terms of a proper time representation for each propagator,

$$
D(k)=\int_{0}^{\infty} d s e^{i s\left(k^{2}-m_{\pi}^{2}+i e\right)}
$$

as

$$
\begin{aligned}
i L(p)= & \int \frac{d^{4} k}{(2 \pi)^{4}} \int_{0}^{\infty} d s_{1} d s_{2} e^{-i\left(s_{1}+s_{2}\right) m_{\pi}^{2}} \\
& \times e^{i s_{1}(p-k)^{2}} e^{i s_{2} k^{2}} .
\end{aligned}
$$

After integrating the Gaussian term in the loop momentum and introducing the variables

$$
s_{1}=s \frac{1-v}{2} \quad \text { and } \quad s_{2}=s \frac{1+v}{2},
$$

we find that the imaginary part, $\Im 2$, is given by

$$
\widetilde{\Im} L=\int_{0}^{1} d v \frac{1}{2 \pi i} \int_{-\infty}^{\infty} \frac{d s}{s} e^{i s\left(\frac{1}{4}\left(1-v^{2}\right) p^{2}-m_{\pi}^{2}\right)} .
$$

Using the integral representation of the Heaviside function, we obtain

$$
\begin{aligned}
\Im L & =\int_{0}^{1} d v \theta\left[\frac{1}{4}\left(1-v^{2}\right) p^{2}-m_{\pi}^{2}\right] \\
& =\sqrt{1-\frac{4 m_{\pi}^{2}}{p^{2}}} \theta\left(p^{2}-4 m_{\pi}^{2}\right),
\end{aligned}
$$

where $p$ is the total momentum that goes into the loop. If we choose $p=\left(2 m_{\pi}, \overrightarrow{0}\right)$, we see that this contribution vanishes. For higher powers in the denominators, which appear when magnetic field terms are introduced, we will use the following identity [22]:

$$
\frac{1}{N !}\left(\frac{i \partial}{\partial \mu^{2}}\right)^{n} \Delta=\Delta^{n+1}
$$

In this way, we found that all the contributions to order $(q B)^{2}$ have the general form

$$
\begin{aligned}
L_{\text {general term }} \propto & (q B)^{2}\left(\frac{\partial}{\partial \mu_{\pi}^{2}}\right)^{2} \\
& \times \int d s_{1} d s_{2} \frac{d^{4} k}{(2 \pi)^{4}} e^{i s_{1}\left(k_{0}^{2}-\vec{k}^{2}-m_{\pi}^{2}+i \epsilon\right)} \\
& \times e^{i s_{2}\left(\left(k_{0}-2 m_{\pi}\right)^{2}-\vec{k}^{2}-\mu_{\pi}^{2}+i \epsilon\right)} .
\end{aligned}
$$

Several of the diagrams that contribute to the $s$ channel have this form where we have to take derivatives with respect to a pion mass parameter, taking then, after the derivation, all masses as the pion mass. Using the identities shown above, we can see that at the threshold these contributions vanish. So, the $s$-channel diagram, calculated at the center-of-mass momentum $p=\left(2 m_{\pi}, \overrightarrow{0}\right)$, does not contribute to the scattering lengths.

Now, we will proceed with the calculation of the equivalent $t$-channel diagram. At the end, we have to also take into account the $u$-channel contribution, which is, however essentially the same as in the $t$ channel. For this calculation, we will invoke some properties of the Hurwitz- $\zeta$ function. If we consider (16), including the coupling, the isospin term, and the magnetic field, integrating the transverse momenta and the proper times, we get

$$
\begin{aligned}
i M_{a, t}= & -\frac{8 \lambda^{4} \pi q B}{(2 \pi)^{4}} \sum_{l=0}^{\infty} \int d k_{0} d k_{3} \\
& \times \frac{(-1)}{\left(q B(2 l+1)-k_{\|}^{2}+m_{\pi}^{2}-i \epsilon\right)^{2}} .
\end{aligned}
$$

In the above expression, $k_{\|}^{2}=k_{0}^{2}-k_{3}^{2}$. Using the mass derivative, and the Plemelj decomposition $(a \pm i \epsilon)^{-1}=$ $\mathrm{P}(1 / a) \mp i \pi \delta(a)$, where $\mathrm{P}$ is the Cauchy principal value, we get

$$
\begin{aligned}
i M_{a, t}= & -\frac{8 \lambda^{4} \pi q B}{(2 \pi)^{4}}\left(\frac{\partial}{\partial m_{\pi}^{2}}\right) \\
& \times \sum_{l=0}^{\infty} \int d k_{0} d k_{3} i \pi \delta\left(q B(2 l+1)-k_{\|}^{2}+m_{\pi}^{2}\right) .
\end{aligned}
$$

After some change of variables, the integration in $k_{0}$ gives us

$$
\begin{aligned}
i M_{a, t}= & -\frac{8 \lambda^{4} \pi^{2}}{(2 \pi)^{4}} i\left(\frac{\partial}{\partial m_{\pi}^{2}}\right) \\
& \times \int d k_{3} \frac{q B}{\sqrt{2 q B}} \zeta\left(\frac{1}{2}, \frac{1}{2}+\frac{k_{3}^{2}+m_{\pi}^{2}}{2 q B}\right),
\end{aligned}
$$

where we have used the Hurwitz- $\zeta$ function

$$
\zeta(s, q)=\sum_{n=0}^{\infty} \frac{1}{(q+n)^{s}} .
$$

We may use the identity [23] 


$$
\zeta(s, a)=\frac{1}{2} a^{-s}+\frac{a^{1-s}}{s-1}+\frac{Z(s, a)}{\Gamma(s)}
$$

where $Z(s, a)$ in the large-a (Poincaré) asymptotic expansion takes the form

$$
Z(s, a) \sim \sum_{k=1}^{\infty} \frac{B_{2 k}}{(2 k) !} \frac{\Gamma(2 k+s-1)}{a^{2 k+s-1}} .
$$

In our case, $a=\frac{1}{2}+\frac{1}{2 x}$, where $x=\frac{q B}{k_{3}^{2}+m_{\pi}^{2}}$. Notice that a large$a$ value corresponds to a small magnetic field. Expanding around $x=0$, we get

$$
\begin{aligned}
i M_{a, t}= & -\frac{8 \lambda^{4} \pi^{2} q B}{(2 \pi)^{4} \sqrt{2 q B}} i\left(\frac{\partial}{\partial m_{\pi}^{2}}\right) \\
& \times \int d k_{3}\left[-\frac{\sqrt{2}}{\sqrt{x}}-\frac{x^{3 / 2}}{12 \sqrt{2}}+O[x]^{7 / 2}\right] .
\end{aligned}
$$

Keeping only the magnetic contribution $(q B)^{2}$, after integrating in $k_{3}$, finally, we find for the $t$-channel contribution

$$
i M_{a, t}=\frac{8 \lambda^{4} \pi^{2}}{(2 \pi)^{4}} i\left(\frac{\partial}{\partial m_{\pi}^{2}}\right)\left(\frac{(q B)^{2}}{12 m_{\pi}^{2}}\right)=-\frac{\lambda^{4} i(q B)^{2}}{24 \pi^{2} m_{\pi}^{4}} .
$$

All the other diagrams reduce to one of the previous cases, once the sigma propagator is cut, i.e., when the approximation $\Delta_{\sigma}\left(k_{0}, \vec{k}, m_{\sigma}\right) \approx-\frac{i}{m_{\sigma}^{2}}$ is used.

After taking all the diagrams into account for the oneloop magnetic corrections to the $\pi-\pi$ scattering amplitudes, we get the amplitude in the $s$ channel,

$$
\begin{aligned}
A_{B}(s, t, u)= & \frac{\left(\frac{1}{4 m_{\pi}^{2}-m_{\sigma}^{2}}\right)^{2}\left(\frac{q B}{m_{\pi}}\right)^{2}\left(\lambda^{6} v^{2}\right)}{4 \pi^{2}} \\
& -\frac{2\left(\frac{q B}{m_{\pi}^{2}}\right)^{2}\left(\lambda^{8} v^{4}\right)}{6 \pi^{2} m_{\sigma}^{4}},
\end{aligned}
$$

and for the $t$ and $u$ channels,

$$
\begin{aligned}
A_{B}(t, s, u)= & A_{B}(u, t, s) \\
= & -\frac{2\left(\frac{q B}{m_{\pi}^{2}}\right)^{2}\left(\lambda^{8} v^{4}\right)}{2 \pi^{2} m_{\sigma}^{4}}-\frac{2\left(\frac{q B}{m_{\pi}^{2}}\right)^{2}\left(\lambda^{8} v^{4}\right)}{6 \pi^{2} m_{\sigma}^{4}} \\
& +\frac{2\left(\frac{q B}{m_{\pi}^{2}}\right)^{2}\left(\lambda^{8} v^{4}\right)}{6 \pi^{2} m_{\sigma}^{4}}-\frac{2\left(\frac{q B}{m_{\pi}^{2}}\right)^{2}\left(5 \lambda^{6} v^{2}\right)}{12 \pi^{2} m_{\sigma}^{4}} \\
& -\frac{2\left(\frac{q B}{m_{\pi}^{2}}\right)^{2}\left(\lambda^{6} v^{2}\right)}{12 \pi^{2} m_{\sigma}^{2}}-\frac{2 \lambda^{4}\left(\frac{q B}{m_{\pi}^{2}}\right)^{2}}{24 \pi^{2}}+\frac{2\left(\frac{q B}{m_{\pi}}\right)^{2}\left(\lambda^{6} v^{2}\right)}{4 \pi^{2} m_{\sigma}^{4}} .
\end{aligned}
$$

\section{RESULTS AND CONCLUSIONS}

The magnetic corrections were calculated analytically. The different parameters in our expressions are renormalized at $B=0$. The linear sigma model, excluding the nucleons, has three parameters: $m_{\pi}^{2}, f_{\pi}$, and $\lambda^{2}$. The first two parameters, $m_{\pi}^{2}$ and $f_{\pi}$, are given by experiments, and the third one is a free parameter. Notice that $f_{\pi}$ is related to the vacuum expectation value $v$. In fact, at tree level, $f_{\pi}=v$. The three parameters are not independent. If instead of $f_{\pi}$ we use the vacuum expectation value $v$ and consider a mass of the sigma meson $m_{\sigma}=700 \mathrm{MeV}$, we have $\lambda^{2}, v=90 \mathrm{MeV}$; if $\lambda^{2}=5.6, v=120 \mathrm{MeV}$ [24]. We know, however, that the mass of the sigma meson is about $m_{\sigma}=550 \mathrm{MeV}$ [25]. Therefore, we need to find new values for $\lambda$ and $v$ associated to the new lower mass of the sigma meson. We found $\lambda^{2}=4.26$ and $v=89 \mathrm{MeV}$, following the philosophy presented in Ref. [24]. The scattering lengths associated to the isospin channels $I=0$ and $I=2$, including our magnetic corrections, are given by

$$
\begin{gathered}
a_{0}^{0}(B)=0.217+\frac{3 A_{B}(s, t, u)+2 A_{B}(t, s, u)}{32 \pi}, \\
a_{0}^{2}(B)=-0.041+\frac{2 A_{B}(t, s, u)}{32 \pi} .
\end{gathered}
$$

The behavior of the normalized scattering lengths $\frac{a_{0}^{0}(B)}{a_{0}^{0}}$ and $\frac{a_{0}^{2}(B)}{a_{0}^{2}}$ is shown in Fig. 4.

The channel $I=2$ corresponds to the most symmetric state for a two-pion state in the isospin space. The fact that the scattering length in this channel increases, due to magnetic effects, shows that the interaction between pions becomes more intense. This, in turn, can be associated to a proximity effect between the pions. In a different context, we have found recently similar effects when computing the

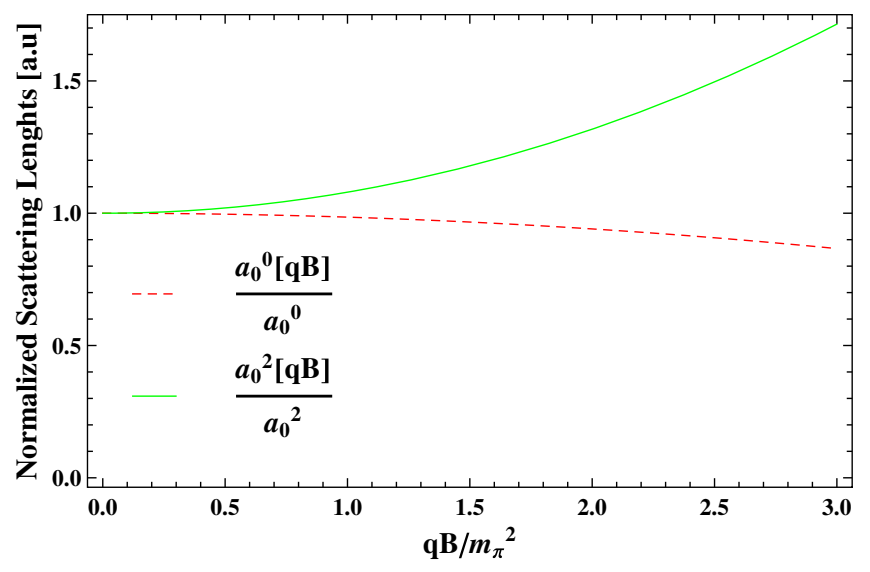

FIG. 4. Scattering lengths normalized to $B=0$. 
correlation distance for quarks in the quark-gluon plasma. This magnitude increases as a function of an increasing external magnetic field [26], with the effect of temperature being exactly the opposite. A similar result was found, this time in the context of QCD sum rules, in which the effects of the magnetic field increase the continuum threshold [27], whereas temperature induces the opposite effect. We see that the results found in this article are consistent with this general picture.

\section{ACKNOWLEDGMENTS}

M. Loewe acknowledges support from FONDECYT (Chile) under Grants No. 1170107, No. 1150471, and No. 1150847 and ConicytPIA/BASAL (Chile) Grant No. FB0821; L. Monje acknowledges support from FONDECYT (Chile) under Grant No. 1170107; and R. Zamora would like to acknowledge support from CONICYT FONDECYT Iniciación under Grant No. 11160234.
[1] L. Rosellet et al., Phys. Rev. D 15, 574 (1977).

[2] B. Adeva et al., Phys. Lett. B 704, 24 (2011).

[3] X.-H. Liu, F.-K. Guo, and E. Epelbaum, Eur. Phys. J. C 73, 2284 (2013).

[4] M. Loewe and J. Ruiz, Phys. Rev. D 78, 096007 (2008).

[5] M. Loewe and C. Martínez, Phys. Rev. D 77, 105006 (2008); 78, 069902 (2008).

[6] A. Ayala, A. Sanchez, G. Piccinelli, and S. Sahu, Phys. Rev. D 71, 023004 (2005).

[7] M. Gell-Mann and M. Lévy, Nuovo Cimento 16, 705 (1960).

[8] C. Contreras and M. Loewe, Int. J. Mod. Phys. A 05, 2297 (1990).

[9] A. Larsen, Z. Phys. C 33, 291 (1986).

[10] N. Bilic and H. Nikolic, Eur. Phys. J. C 6, 515 (1999).

[11] H. Mao, N. Petropoulos, and W.-K. Zhao, J. Phys. G 32, 2187 (2006); N. Petropoulos, arXiv:hep-ph/0402136 and references therein.

[12] B. J. Schaefer and M. Wagner, Phys. Rev. D 79, 014018 (2009).

[13] P. Kovacs and Z. Szep, Phys. Rev. D 77, 065016 (2008).

[14] P. Kovacs and Z. Szep, Phys. Rev. D 75, 025015 (2007).

[15] P. Kovacs and Z. Szep, Phys. Rev. D 93, 114014 (2016).

[16] P. D. B. Collins, An Introduction to Regge Theory on High Energy Physics (Cambridge University Press, Cambridge, England, 1977).
[17] S. S. M. Wong, Introductory Nuclear Physics (Wiley, New York, 1998).

[18] J. Gasser and H. Leutwyler, Ann. Phys. (Berlin) 158, 142 (1984).

[19] B. Peyaud, Nucl. Phys. B, Proc. Suppl. 187, 29 (2009).

[20] S. Weinberg, Phys. Rev. Lett. 17, 336 (1966).

[21] G. Piccinelli and A. Sánchez, Phys. Rev. D 96, 076014 (2017).

[22] Y. Fujimoto, H. Matsumoto, and H. Umezawa, Phys. Rev. D 30, 1400 (1984).

[23] W. Magnus, F. Oberhettinger, and R. P. Soni, Formulas and Theorems for the Special Functions of Mathematical Physics (Springer, Berlin, 1966).

[24] J. L. Basdevant and B. W. Lee, Phys. Rev. D 2, 1680 (1970).

[25] E. M. Aitala et al., Phys. Rev. Lett. 86, 765 (2001); 86, 770 (2001); J. M. de Miranda and I. Bediaga, in Proceedings of the Eleventh International Conference on Hadron Spectroscopy, (AIP, New York, 2006), Vol. 814.

[26] A. Ayala, L. A. Hernandez, M. Loewe, A. Raya, J. C. Rojas, and R. Zamora, Phys. Rev. D 96, 034007 (2017).

[27] A. Ayala, C. A. Dominguez, L. A. Hernandez, M. Loewe, J. C. Rojas, and C. Villavicencio:, Phys. Rev. D 92, 016006 (2015). 